\title{
Nuclear effects in leptoproduction of secondaries
}

\author{
Ya.A. Berdnikov, ${ }^{1}$ M.M. Ryzhinskiy,,$^{1, *}$ and Yu.M. Shabelski ${ }^{2, * *}$ \\ ${ }^{1}$ St.-Petersburg State Polytechnic University, St.-Petersburg, Russia \\ ${ }^{2}$ Petersburg Nuclear Physics Institute, Gatchina, Russia
}

\begin{abstract}
We estimate the energy losses in the case of deep-inelastic scattering on nuclear targets in terms of the effective change of the virtual photon energy. Our phenomenological results are in reasonable agreement with theoretical calculations. The difference in secondary production processes in hard and soft interactions is discussed.
\end{abstract}

PACS numbers: 25.30.-c, 25.75.Dw, 13.87.Fh

\section{INTRODUCTION}

The inclusive spectra of secondaries (pions, kaons, $p$ and $\bar{p}$ ) measured in lepton-nucleus (lA) deep-inelastic scattering (DIS) [1-3] become more and more soft with the increase of atomic weight of the target. In this paper we consider the $A$ dependences of these spectra using the same method as in our previous paper [4].

In the case of DIS only some part of the projectile lepton energy $\left(\nu=E_{l}-E_{l}^{\prime}\right)$ is transfered to the target. Thus, one can consider the virtual photon as the projectile to draw an analogy between the DIS and hadroproduction processes. It is convenient to analyze DIS data in terms of energy fractions of the virtual photon carried by the produced hadron. Therefore in this paper we will use the variable:

$$
z=\frac{E_{h}}{\nu}
$$

where $E_{h}$ is the energy of the produced hadron in the lab. frame.

At high energies in the case of nucleon target the maximum value of $z$ is close to unity $\left(z_{\max } \rightarrow 1\right)$. In the case of nuclear target the situation is more complicated because there are many different contributions $[5,6]$ from the final-state interactions with nuclear matter

\footnotetext{
* Electronic address: mryzhinskiy@phmf .spbstu.ru

** Electronic address: shabelsk@thd.pnpi.spb.ru
} 
which decrease the spectra at large $z$. On the other hand, the processes which leads to the so-called cumulative effect [7-9] increase the boundaries of the available $z$ region ${ }^{1}$. The modification of parton structure function [10] should be accounted for.

In all processes on nuclear targets (except of the coherent ones) some fraction of energy is used for nuclear disintegration. The nuclear target is destroyed and several nucleons, as well as light nuclear fragments, (say, $\alpha$ particles) appear in the final state. It can be considered as a phase-space limitation for the produced secondaries. The corresponding fraction of initial energy used on this effect numerically is not so small (it is many times larger than the nuclear binding energy).

The energy used for nuclear disintegration is taken from the beam or secondary particle energy, primarely via some QCD process, discussed in [5, 6]. After several stages this energy transforms (in part, as a minimum) into kinetic energy of the target fragments. So the portion of the last kinetic energy allows us to estimate the primary energy losses all together. On the other hand, the phase-space limitation can be considered as an effective decrease of the incident beam energy.

In what follows we will consider the $A$ dependences of secondary hadron leptoproduction in terms of $z_{A}$ :

$$
z_{A}=\frac{E_{h}}{\nu-E_{A}}
$$

and we assume that it is possible to find the shift $E_{A}$ for the case of interaction with nuclear target from the condition that the ratios of secondary multiplicities on nuclear and nucleon $\left(E_{A=1}=0\right)$ targets in terms of $z_{A}$

$$
R_{l A / l p}\left(z_{A}\right)=\operatorname{const}\left(z_{A}\right) \simeq 1
$$

whereas the same ratios in terms of $z$

$$
R_{l A / l p}(z)=f(z)
$$

Evidently, such rescale is reasonable only for not very small $z$ values.

We will determine shifts $E_{A}$ from the experimental data and we will compare them with several independent estimations. Such approach allows us, in particular, to investigate the

1 The last processes have rather small cross section. 
energy $(\nu)$ dependence of all nuclear effects. In conclusion we will compare our results with theoretical calculations [5, 6].

\section{2. $A$ DEPENDENCE OF SECONDARY LEPTOPRODUCTION AT}

\section{LARGE $Z_{A}$}

The experimental results for semi-inclusive deep-inelastic scattering on nuclei are usually presented in terms of the hadron multiplicity ratio $R_{A / D}$, which represents the ratio of the number of hadrons of type $h$ produced per deep-inelastic scattering event on a nuclear target of mass $A$ to that from a deuterium target $(D)$. The multiplicity ratio is defined as:

$$
R_{A / D}=\frac{\frac{1}{A}\left(\frac{d \sigma}{d z}\right)_{l A \rightarrow h}}{\frac{1}{2}\left(\frac{d \sigma}{d z}\right)_{l D \rightarrow h}},
$$

where $\left(\frac{d \sigma}{d z}\right)_{l A \rightarrow h}$ is the yield of semi-inclusive hadrons $h$ from the nucleus $A$ in a given $z$-bin.

In this section we are going to analyze the ratio Eq. (5) in the following way. Suppose one has two $z$ spectra for DIS on nucleon and nuclear targets or on two different nuclei (light and heavy ones). Then one can shift the spectrum that corresponds to the heavy nucleus according to Eq. (2) by changing $E_{A}$ parameter. Assuming that nuclear effects are small for very light nuclei, it is possible then to calculate the fraction of the virtual photon energy spent on nuclear effects. This may be done by calculating the ratio of the shifted spectrum to the spectrum that corresponds to the light nucleus. When this ratio is close to unity then the corresponding shift will give the absolute value of energy loss caused by the mentioned nuclear effects.

For the purpose of our analysis we used the experimental data on DIS on nuclei measured by the HERMES Collaboraton [1, 2] as well as EMC data [3].

The HERMES results for deep-inelastic $e^{+} \mathrm{D}$ and $e^{+} \mathrm{Kr}$ scattering at $27.5 \mathrm{GeV}$ are available in terms of the ratio Eq. (5) for different set of secondaries (pions, kaons, $p$ and $\bar{p}$ ) [1]. Neutral pion and averaged charged pion multiplicities for DIS of positrons on hydrogen at the same energy are published in Ref. [2]. Neglecting the difference between hydrogen and deuterium targets one can extract the multiplicity for pions produced on a heavy target ( $\mathrm{Kr}$ ) from the multiplicity ratio and absolute multiplicity spectrum for hydrogen. The measured ratio for neutral pions is presented in Fig. 1a. In Fig. 1b one can see extracted spectrum for Kr target 
(solid circles) as well as neutral pion multiplicity for hydrogen (open circles) from Ref. [2]. One can see evident suppression of Kr spectrum in comparison with hydrogen one.

Having two multiplicity spectra for $p$ and $\mathrm{Kr}$ targets one can analyze them in the way described above. Fig. 2 represents the shifted $\mathrm{Kr}$ spectrum with $E_{A}=1 \mathrm{GeV}$ (solid circles) as well as spectrum for hydrogen measured by HERMES (open circles).

Now to estimate how much energy of the virtual photon is spent on nuclear effects one should calculate the ratio Eq. (5) of the shifted Kr spectrum to the hydrogen spectrum. The calculated ratios are presented in Fig. 3 for different $E_{A}$ values (squares). The figure also represents original ratio without any shift (solid circles). One can see that corresponding values of energy losses lie between $E_{A}=1.0$ and $1.4 \mathrm{GeV}$.

Omitting intermediate calculations we present results of the analysis for charged pions (from the same experiment) at the same energies (see Fig. 4a for $\pi^{+}$and Fig. 4b for $\pi^{-}$). From the last two figures (Fig. 3 and 4) one can see approximately the same suppression for all pions.

There are additional data on the market relevant for such an analysis, namely the data on deep-inelastic $\mu \mathrm{D}$ and $\mu \mathrm{Cu}$ scattering at $100-280 \mathrm{GeV}$ obtained by EMC [3]. They measured differential multiplicities of forward produced charged hadrons on both nuclei $(\langle\nu\rangle=60 \mathrm{GeV})$, which can be used directly in our analysis as described above. The results of the analysis one can see in Fig. 5. Fig. 5a represents multiplicities for D (solid circles) and $\mathrm{Cu}$ (open circles) targets measured by EMC, and Fig. 5b represents the ratios obtained with our analysis (squares) as well as the original EMC ratio (solid circles).

Results for energy losses obtained for $\mathrm{Cu}$ target $E_{A} \approx 1.4 \mathrm{GeV}$ are in reasonable agreement with those obtained for $\mathrm{Kr}$ target. However, HERMES measurements were done at $\langle\nu\rangle \approx$ $16 \mathrm{GeV}$, while EMC data were taken at $\langle\nu\rangle \approx 60 \mathrm{GeV}$. Therefore one can conclude that energy dependence of nuclear effects for all charged particles here is rather weak within the errors of the analysis.

Unfortunately the inclusive spectra of identified secondaries are published only at one (HERMES) energy and we cannot discuss the energy dependence of our $E_{A}$ parameter. However, there exist the experimental results [1] for $R_{A / D}$ as a function of $\nu$. For pions these ratios increase with $\nu$ and it means that $E_{A}$ values have at least more weak $\nu$ dependence than the linear one. The values of $R_{A / D}$ are evidently different for secondaries $K^{+}$and $K^{-}$, as well as for $p$ and $\bar{p}$. Principally, this difference can be connected [11] with different 
absorption cross sections of secondaries. Another possible explanation for evident difference in yields of secondary $p$ and $\bar{p}$ comes from the baryon charge diffusion from the target to forward hemisphere due to string junction mechanism [12-15].

\section{DIFFERENCE BETWEEN THE SECONDARY PRODUCTION IN HARD AND SOFT PROCESSES}

Let us try to use the presented approach, see also [4], for the description of $A$ dependences of the spectra of secondaries produced in soft hadron nucleus collisions.

Let us define the variable

$$
x_{0}=\frac{p}{p_{0}},
$$

where $p$ is the momentum of the produced secondary and $p_{0}$ is the initial momentum of the beam particle, both in c.m. frame, and the variable

$$
x_{\mathrm{F}}=\frac{p}{p_{0}-p_{A}}
$$

where the shift $p_{A}$ accounts for all nuclear effects.

Let us define the ratio of the multiplicities of secondary hadrons of type $h$ produced in $h_{1} A$ and $h_{1} p$ collisions as

$$
R_{A / p}(x)=\frac{\frac{1}{A}\left(\frac{d \sigma}{d x}\right)_{h_{1} A \rightarrow h}}{\left(\frac{d \sigma}{d x}\right)_{h_{1} p \rightarrow h}},
$$

Similarly to Eqs. (3) and (4) we assume that

$$
R_{A / p}\left(x_{\mathrm{F}}\right)=\operatorname{const}\left(x_{\mathrm{F}}\right) \simeq 1
$$

whereas the same ratios in terms of $x_{0}$ depends on $x_{0}$

$$
R_{A / p}\left(x_{0}\right)=f\left(x_{0}\right)
$$

Such assumption leads to the agreement [4] with the data on $J / \Psi$ and Drell-Yan pair production on nuclear targets with $p_{A} \ll p_{0}$. Now we use the same assumption for the case of soft $\Lambda$ production in $p A$ collisions, and the results for the data [16] are shown in Fig. 6. We can see total disagreement with the data. The values of $R_{A / p}\left(x_{\mathrm{F}}\right)$ depend very weakly on $p_{A}$ value and we can not find such $p_{A}$ value when Eq. (9) is fulfilled. 
The data for $p A \rightarrow p X$ at small $p_{T}$ [17] show the behaviour similar to the case shown in Fig. 6, whereas the situation with the data on soft pion production in $p A$ and $\pi A$ collisions [17] is rather unclear due to the large experimental error bars.

We can try to explain the presented difference in nuclear effects for hard and soft production of secondaries by the essential difference in impact parameters which give the main contribution in these two cases.

Let us consider the $A$ dependences of the inclusive production cross section as

$$
x_{\mathrm{F}} d \sigma / d x_{\mathrm{F}} \sim A^{\alpha\left(x_{\mathrm{F}}\right)}
$$

In hard collisions $\alpha\left(x_{\mathrm{F}}\right) \sim 1$ except of rather large $x_{\mathrm{F}}$ values. It means that the considered secondary particle can be produced on every target nucleon with equal probability, i.e. absorption effects are small. Even for the secondaries produced at large $x_{\mathrm{F}}, \alpha\left(x_{\mathrm{F}}\right)>2 / 3$. So all impact parameters contribute here, and some decrease of $\alpha$ values at large $x_{\mathrm{F}}$ can be connected with the effective decrease of the beam energy, as it was shown in [4] and in Section 2 of the present paper.

In the case of soft production of secondaries the experimental values of $\alpha$ at $x_{\mathrm{F}}>0.2-0.3$ are about $1 / 3$ and, contrary to the case of hard production, they rather weakly depend on $x_{\mathrm{F}}$ in this $x_{\mathrm{F}}$ region. This corresponds to the picture when the secondaries at $x_{\mathrm{F}}>0.2-0.3$ are produced mainly in one-fold interaction of beam particle with the target nucleus. The corresponding cross section for $\nu$-fold inelastic interactions has the form $[18,19]$

$$
\sigma_{\text {prod }}^{(\nu)}=\frac{1}{\nu !} \int d^{2} b\left[\sigma_{i n} T(b)\right]^{\nu} e^{-\sigma_{i n} T(b)},
$$

where $\sigma_{i n}$ is the inelastic interaction cross section of beam particle with a nucleon. For hadron beam the product $\sigma_{i n} T(b)$ is small enough only for large impact parameters. This leads immediately to the value $\alpha \sim 1 / 3$ for large $x_{\mathrm{F}}$ where multiple interactions $(\nu>1)$ can not contribute due to the energy conservation. The ratio Eq. (8) is determined now by the ratio $\sigma_{\text {prod }}^{(1)} / \sigma_{\text {prod }}\left(\sigma_{\text {prod }}=\sum_{\nu=1}^{A} \sigma_{\text {prod }}^{(\nu)}\right)$.

The behaviour of $\Lambda$ yields produced in hard and soft $p A$ to $p p$ collisions, calculated in the framework of the Quark-Gluon String Model [20, 21] is shown in Fig. 7. The difference in the soft and hard interactions is reproduced rather clear. In the case of soft interactions we obtain rather strong $x_{\mathrm{F}}$-dependence of $\alpha\left(x_{\mathrm{F}}\right)$ Eq. (11) at small $x_{\mathrm{F}}$ and rather weak dependence at large $x_{\mathrm{F}}$. The case of "hard production" was generated with the help of rescale, Eq. (7), 
assuming firstly $\alpha\left(x_{\mathrm{F}}\right)=1$. Here we obtain the behaviour typical for experimental hard production, weak $x_{\mathrm{F}}$-dependence of $\alpha\left(x_{\mathrm{F}}\right)$ at small $x_{\mathrm{F}}$ and strong dependence at large $x_{\mathrm{F}}$.

\section{CONCLUSION}

In summary, we considered energy losses of the virtual photon in deep-inelastic $l A$ collisions from the available experimental data. What we were interested in is how much energy of the virtual photon is spent on all nuclear effects including the effect of nuclear disintegration.

Our results for energy losses $E_{A} \approx 1.4 \mathrm{GeV}$ obtained for $\mathrm{Kr}$ and $\mathrm{Cu}$ targets correspond to energy loss ratios $d E / d z=0.5-0.6 \mathrm{GeV} / \mathrm{fm}$ (we assumed that DIS takes place somewhere in the center of the nucleus). This value is $\approx 5$ times smaller than we obtained in our previous analysis [4]. However, in the present paper we deal with energies many times smaller than in the previous one. From comparison between HERMES and EMC data obtained at different virtual photon energies ( $\nu=7-12 \mathrm{GeV}$ and $60 \mathrm{GeV}$ respectively) we conclude that our $E_{A}$ value, within the accuracy of the performed analysis, has very weak $\nu$-dependence.

There are a lot of models for leptoproduction of hadrons in nuclear DIS. They use different approaches such as absorption of a prehadron state and modification of quark fragmentation functions in nuclei [6, 22], energy losses (vacuum and induced), production and formation times of a prehadron and a hadron respectively [5]. There is an experimental evidence for the effect of nuclear modification of nucleon structure functions [10], thus, all the models must take into account this effect as well. The obtained results for HERMES data are in reasonable agreement with the theoretical calculations of Refs. [5, 6], since both cited models agree well with the HERMES data on pion production. Concerning our result obtained for EMC, in [5] these data $(\nu=60 \mathrm{GeV})$ were not considered. However, in Ref. [6] it was shown that the effect of absorption of the prehadron is negligible for these data, which implies that the main contribution to the observed suppression comes from the effect of nuclear modification of quark fragmentation functions, while in the case of HERMES data both the effects are important.

However, concluding that some or other effect (if there is no experimental evidence for it) gives the main contribution to the considering process is questionable. E.g. if one consideres models for energy loss and absorption, they both lead to a suppression law $\sim A^{2 / 3}$ (broken at 
$\mathrm{A} \geq 80$ ), thus, one cannot completely distinguish between these two effects [23]. Actually this does not allow us do draw a definite conclusion which effect is dominant in the considered processes of nuclear DIS for HERMES and EMC. The only we can say is how much energy of the virtual photon is spent on all nuclear effects. Obviously, among the others there exist two effects: nuclear disintegration and modification of nuclear structure functions (however, they are not dominant here).

In section 3 we have demonstrated that one could not apply our analysis to soft processes. We also put forward an argument for this fact which lies in that in the case of hard processes every nucleon gives an equal contribution to the secondary production cross section (because $d \sigma / d x \sim A^{\alpha}$, where $\alpha \sim 1$ ), while in the case of soft processes only a rather small part of all the nucleons contribute to the cross section (because $\alpha \sim 1 / 3$ ). Moreover, these contributing nucleons are concentrated on the peripherals of the nucleus. The number of these nucleons is not changed with $x_{\mathrm{F}}$ at large $x_{\mathrm{F}}$, so the ratio of secondary yields on nuclear and nucleon targets is practically constant.

We are grateful to M. G. Ryskin for discussions.

1. A. Airapetian et al. (HERMES Collab.), Phys. Lett. B577, 37 (2003), hep-ex/0307023.

2. A. Airapetian et al. (HERMES Collab.), Eur. Phys. J. C21, 599 (2001), hep-ex/0104004.

3. J. Ashman et al. (EMC Collab.), Z. Phys. C52, 1 (1991).

4. Ya. A. Berdnikov, M. M. Ryzhinskiy and Yu. M. Shabelski, Yad. Fiz. (in print), hep$\mathrm{ph} / 0510152$.

5. B. Z. Kopeliovich et al., Nucl. Phys. A740, 211 (2004), hep-ph/0311220.

6. A. Accardi et al., Nucl. Phys. A720, 131 (2003), nucl-th/0211011.

7. A. M. Baldin et al., Yad. Fiz. 18, 79 (1973).

8. Yu. D. Bayukov et al., Yad. Fiz. 18, 1246 (1973).

9. L. L. Frankfurt and M. L. Strikman, Phys. Rept. 76, 215 (1981).

10. K. J. Eskola, Nucl. Phys. B400. 240 (1993).

11. A. Bialas, Z. Phys. C26, 301 (1984).

12. D. Kharzeev, Phys. Lett B378, 238 (1996).

13. B. Z. Kopeliovich and B. Povh, Z. Phys. C75, 693 (1997). 
14. G. H. Arakelyan et al., Eur. Phys. J. C26, 81 (2002).

15. F. Bopp and Yu. M. Shabelski, Yad. Fiz. 68, 2155 (2005).

16. P. Scubic et al., Phys. Rev. D18, 3115 (1978).

17. D. S. Barton et al., Phys. Rev. D27, 2580 (1983).

18. J. S. Trefil and F. von Hippil, Phys. Rev. D7, 2000 (1973).

19. Yu. M. Shabelski, Yad. Fiz. 26, 1084 (1977); Nucl. Phys. B132, 491 (1978).

20. A. B. Kaidalov and K. A. Ter-Martirosyan, Yad. Fiz. 39, 1545 (1984); 40, 211 (1984).

21. Yu. M. Shabelski, Yad. Fiz. 45, 223 (1987).

22. N. Armesto et al. Phys. Lett. B366, 276 (1996); Yad. Fiz 61, 134 (1998).

23. A. Accardi, nucl-th/0510090. 

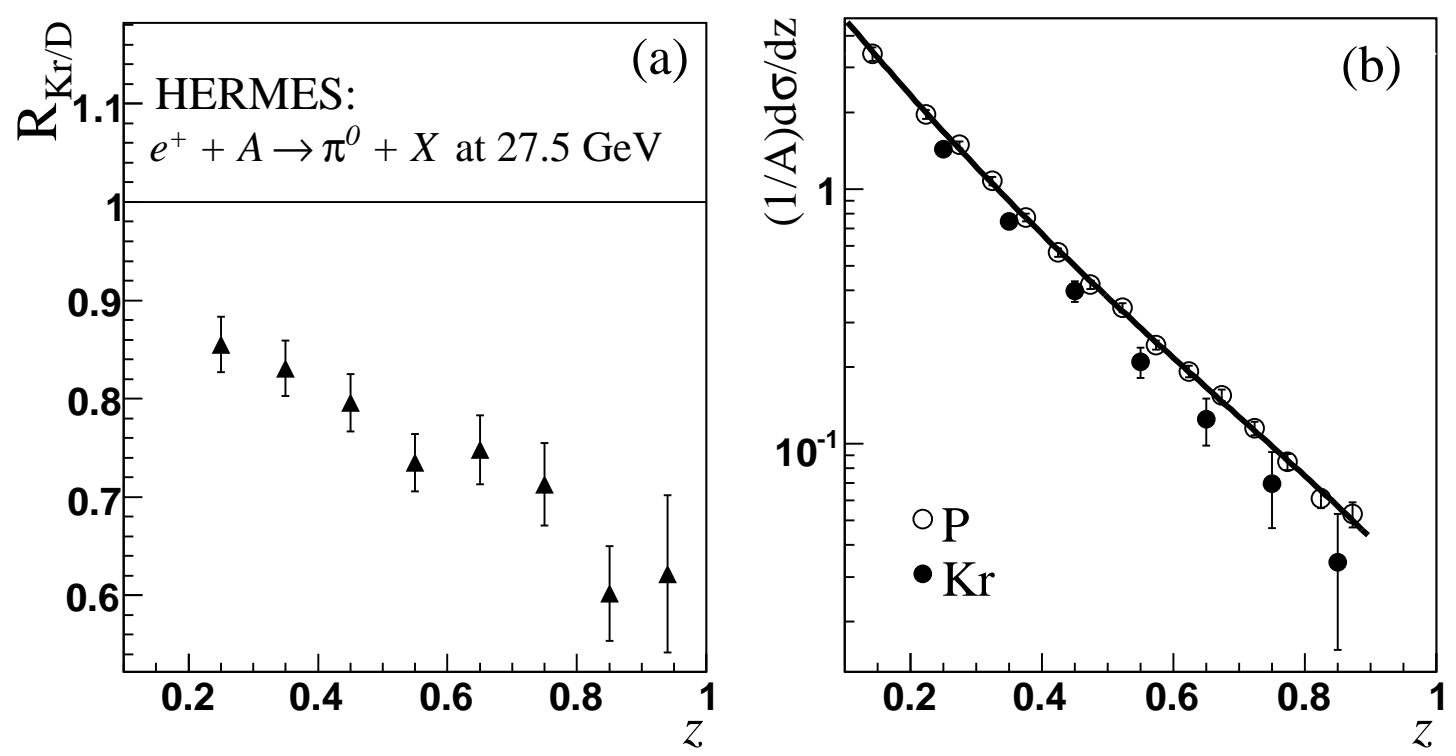

Figure 1. (a) Multiplicity ratio for identified neutral pions from a $\mathrm{Kr}$ target as a function of $\mathrm{z}$ (for $\nu>7 \mathrm{GeV}$ ). (b) Neutral pion multiplicities for hydrogen [2] and $\mathrm{Kr}$ (extracted from ratio Fig. 1a). 


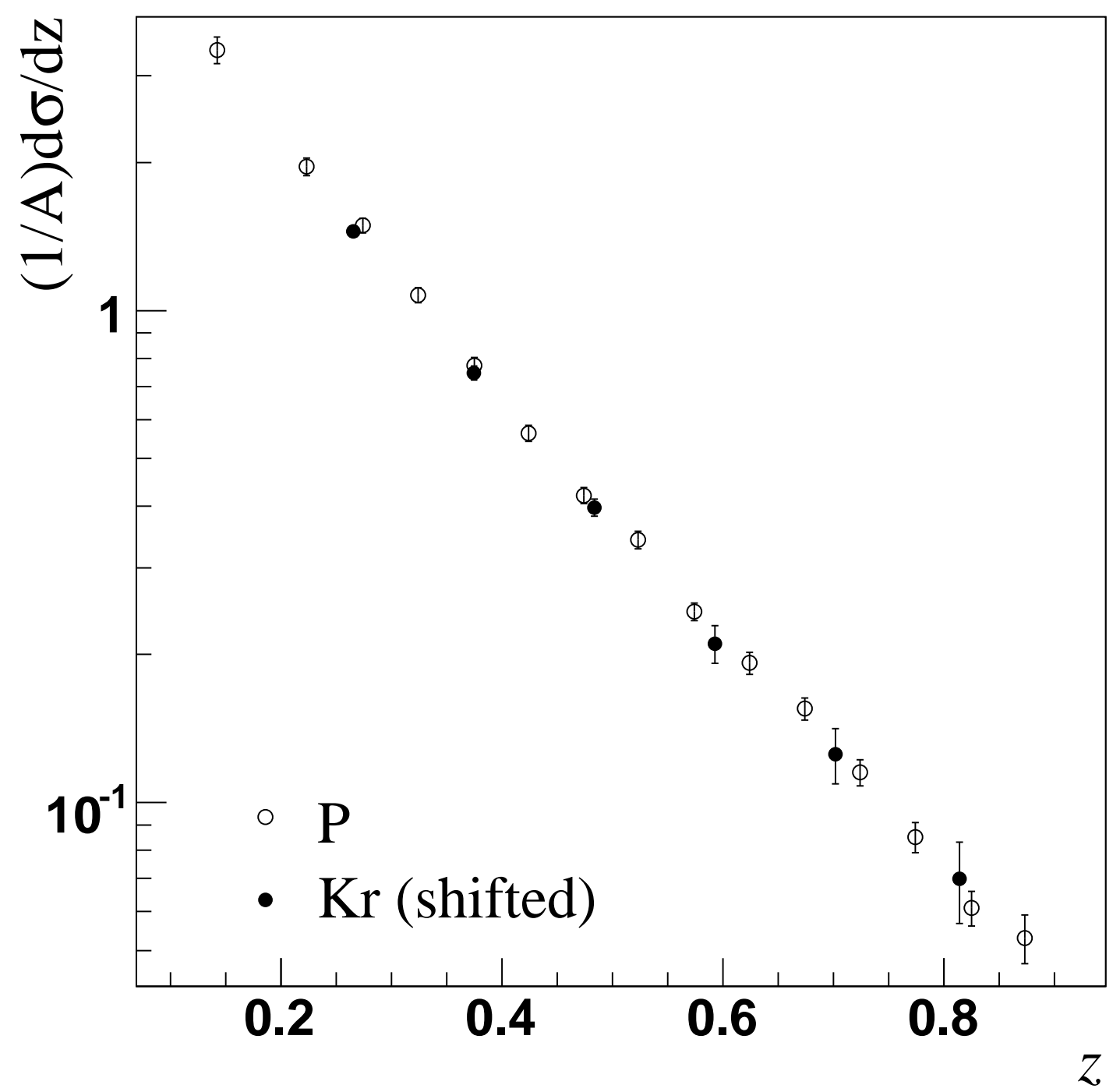

Figure 2. The same as in Fig. 1b, but the spectrum for $\mathrm{Kr}$ target was shifted according to Eq. (7) with $E_{A}=1.0 \mathrm{GeV}$. 


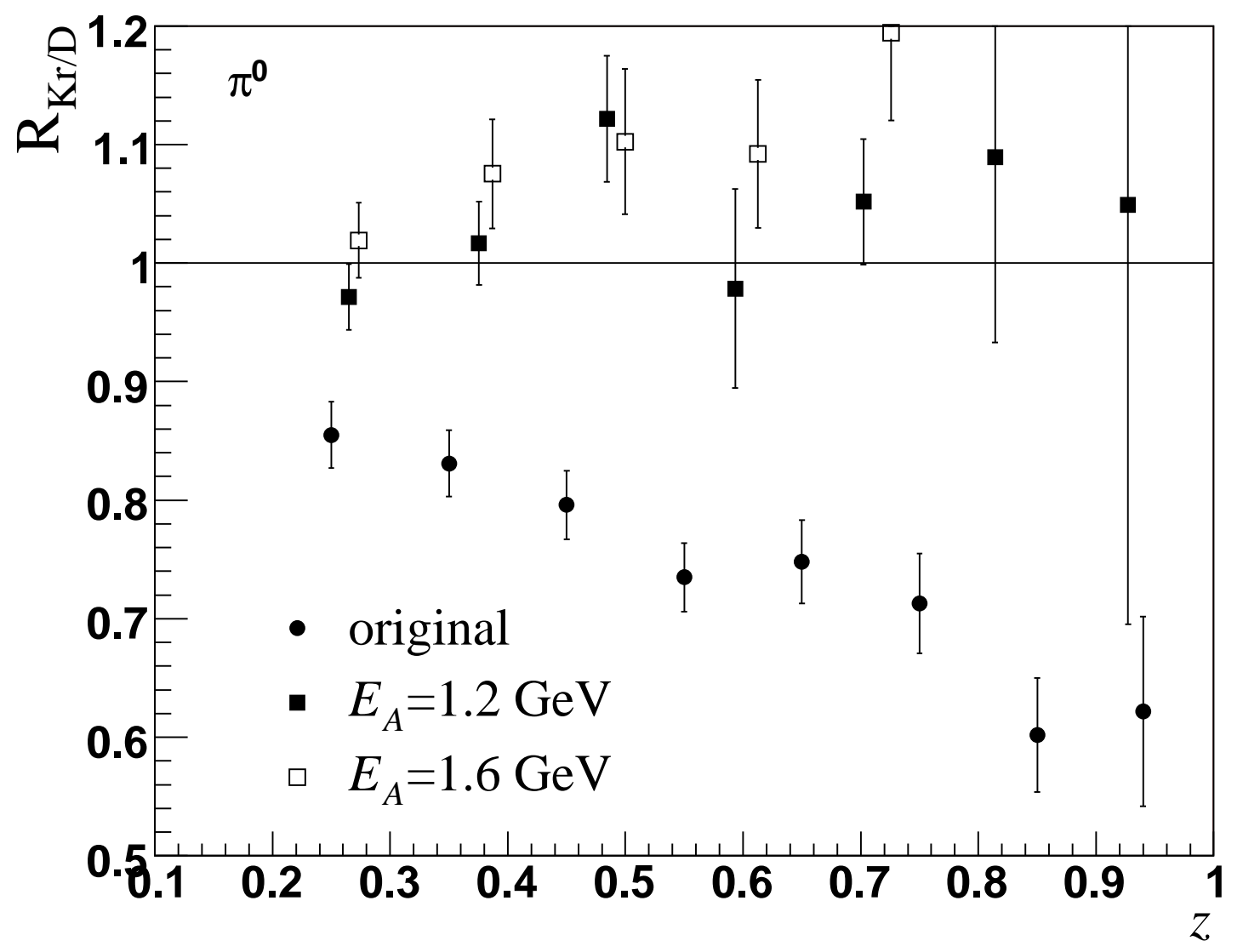

Figure 3. Multiplicity ratios for neutal pions from $\mathrm{Kr}$ target: original ratio measured by HERMES [1] (solid circles), shifted ratios at $E_{A}=1.2 \mathrm{GeV}$ (solid squares) and $E_{A}=1.6 \mathrm{GeV}$ (open squares). 

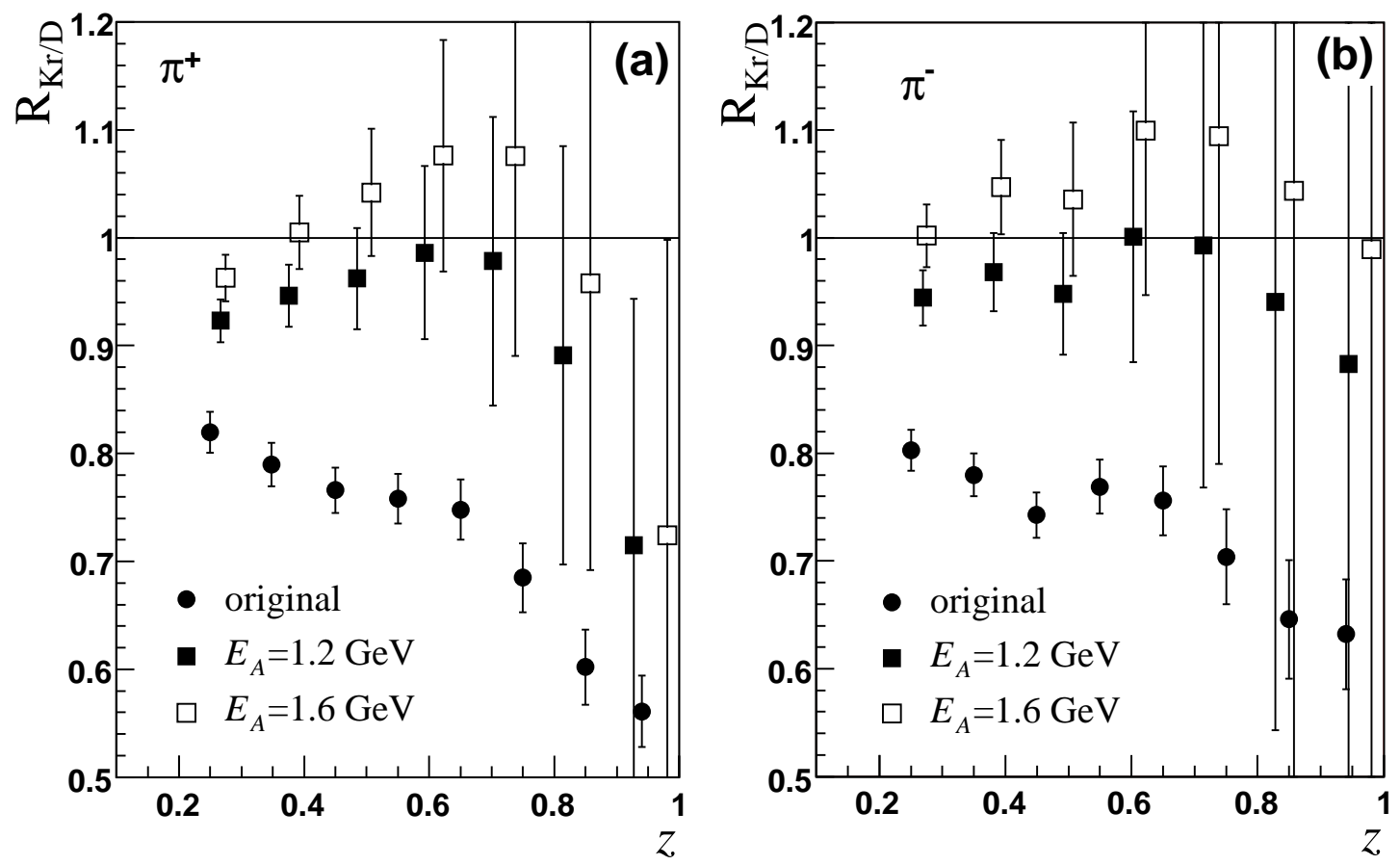

Figure 4. Multiplicity ratios for (a) $\pi^{+}$: original ratio measured by HERMES [1] (solid circles), shifted ratios at $E_{A}=1.2 \mathrm{GeV}$ (solid squares) and $E_{A}=1.6 \mathrm{GeV}$ (open squares); (b) $\pi^{-}$: original ratio measured by HERMES [1] (solid circles), shifted ratios at $E_{A}=1.2 \mathrm{GeV}$ (solid squares) and $E_{A}=1.6 \mathrm{GeV}$ (open squares). 

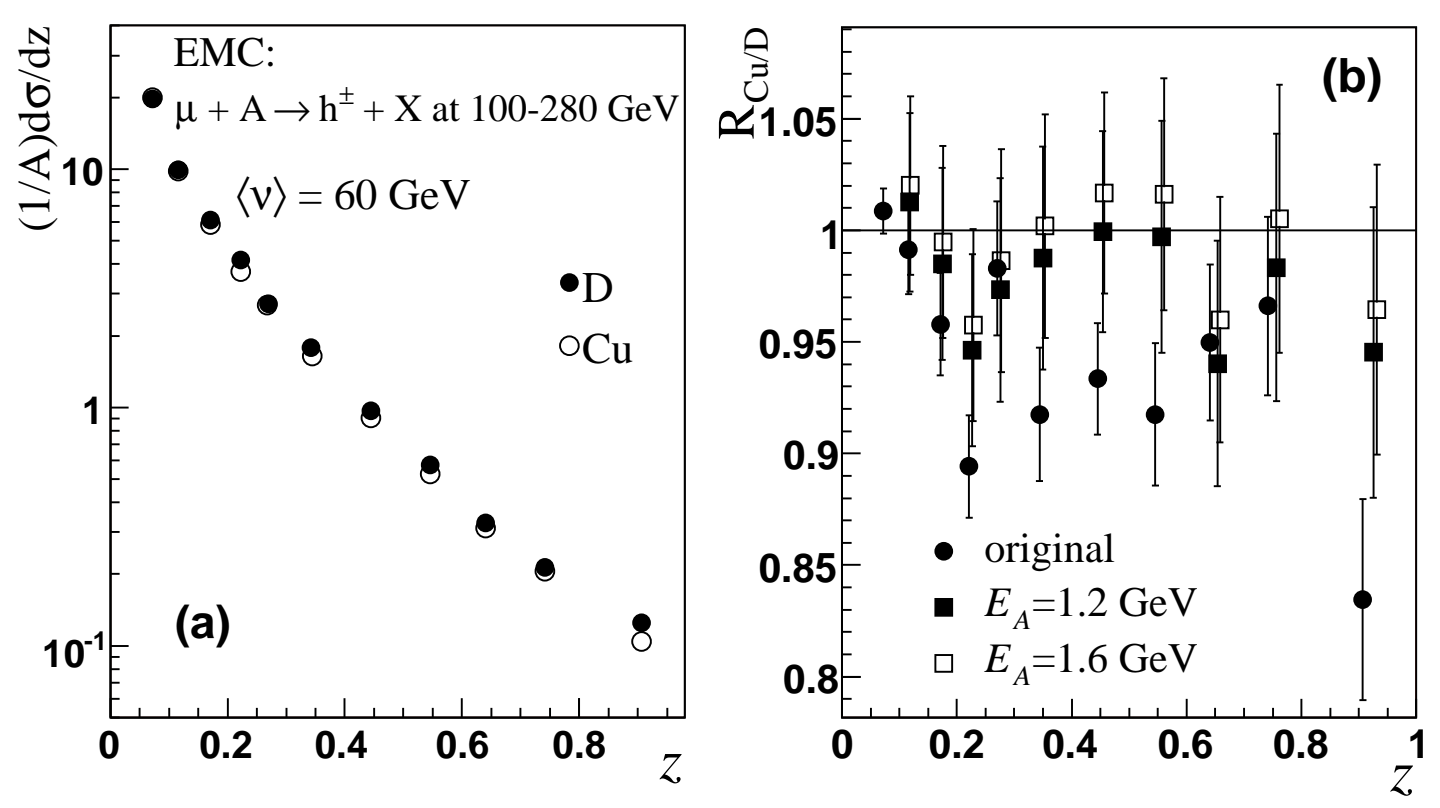

Figure 5. (a) Differential hadron multiplicity as a function of $z$ for $\mathrm{Cu}$ (open circles) and $\mathrm{D}$ (solid circles). The statistical errors are of a similar size to the symbols, the systematic errors are not shown. (b) Multiplicity ratios for hadrons from $\mathrm{Cu}$ target: original ratio measured by EMC [3] (solid circles), shifted ratios at $E_{A}=1.2 \mathrm{GeV}$ (solid squares) and $E_{A}=1.6 \mathrm{GeV}$ (open squares).
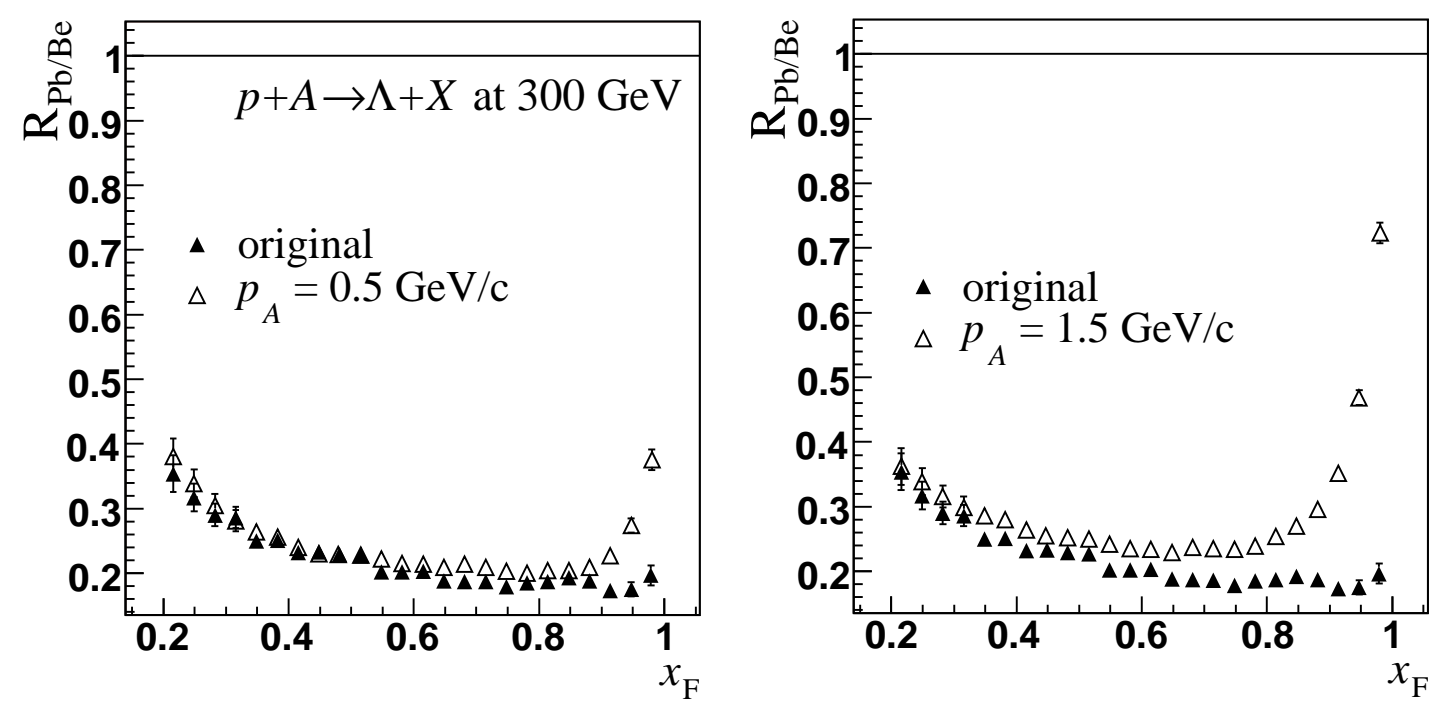

Figure 6. Ratios of the multiplicities of $\Lambda$ produced softly $(\theta=0.25 \operatorname{mrad}) \quad$ on $\mathrm{Pb}$ and $\mathrm{Be}$ targets at $300 \mathrm{GeV}$. 


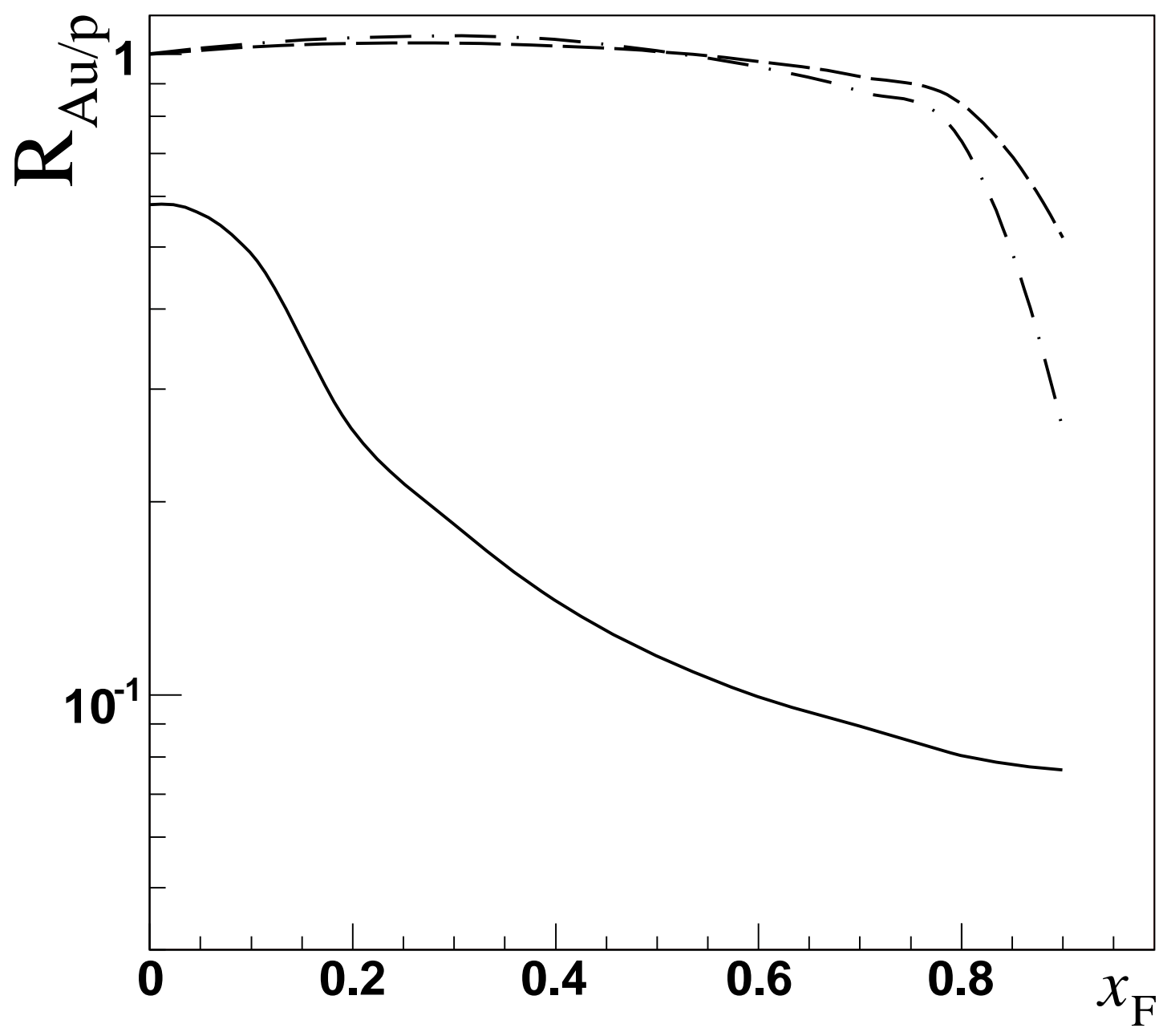

Figure 7. The calculated ratios of the multiplicities of $\Lambda$ produced in hard and soft $p A$ and $p p$ collisions at $\sqrt{s}=20 \mathrm{GeV}$. The curves for "hard" production were obtained with $p_{A}=0.5 \mathrm{GeV} / \mathrm{c}$ (dashed curve) and $p_{A}=0.75 \mathrm{GeV} / \mathrm{c}$ (dash-dotted curve). 